\title{
A Simple Mathematical Approach to Human Society
}

Miklos Szilagyi

DAIMI PB - 261

August 1988 
A SIMPLE MATHEMATICAL APPROACH TO HUMAN SOCIETY

Miklos Szilagyi

Department of Computer Science, Aarhus University, Aarhus, Denmark (Permanent address: Miklos Szilagyi,

Professor of Electrical and Computer Engineering,

University of Arizona, Tucson, AZ 85721)

Abstract - A model is proposed for the treatment of human society as a system of highly interconnected units whose behavior is described by a system of coupled differential equations. The stable solutions of this system represent stable formations in society. The concepts of 'social energy' and 'social temperature' are introduced for the description of these stable formations as energy minima. Changes in society are explained as redistributions of the connections between the units. Some simple relationships between political parties are analyzed as examples. The model can be used at different levels, and it can provide help both for the analysis of past political events and for the development of future political strategies. 


\section{A SIMPLE MATHEMATICAL APPROACH TO HUMAN SOCIETY}

Abstract - A model is proposed for the treatment of human society as a system of highly interconnected units whose behavior is described by a system of coupled differential equations. The stable solutions of this system represent stable formations in society. The concepts of 'social energy' and 'social temperature' are introduced for the description of these stable formations as energy minima. Changes in society are explained as redistributions of the connections between the units. Some simple relationships between political parties are analyzed as examples. The model can be used at different levels, and it can provide help both for the analysis of past political events and for the development of future political strategies.

\section{INTRODUCTION}

The enormous complexity of the real world can be dealt with by using simplified and idealized models that abstract its essential properties. The relationships between model objects can always be expressed in mathematical terms. Simulation (and particularly computer simulation) is experimentation with such models. Computer simulation is convenient for experiments which might be slow, expensive, dangerous or impossible with real-world systems (Korn, 1988). Although it is usually easier to express social phenomena in terms of integers, sometimes it is more convenient to model them as continuous, differentiable variables. Then their behavior can easily be described by means of differential equations. This approach is commonly used for the description of social phenomena. For example, a population dynamics model can provide insight into epidemic propagation, a competitive dynamical system model can describe the voting paradox or a globally 
stable economic market (Grossberg, 1988). There has even been an attempt to describe "the entire world" in terms of some specially selected variables (Forrester, 1972).

In this paper we shall assume that the human society is a system of highly interconnected units whose behavior is described by a system of coupled differential equations. Depending on the level of the model, these units can represent nations, political parties, special interest groups or even individuals. The stable solutions of the system of differential equations correspond to stable formations in society. The concepts of 'social energy' and 'social temperature' will be introduced for the description of these stable formations as energy minima. Changes in society will be explained as redistributions of the connections between the units that take place more or less rapidly according to different 'temperatures'. The role of the connections between the units of the system will be shown on concrete simulation examples. The model can be used both for the analysis of past political events and for the development of future political strategies.

\section{THE MODEL}

Let us now consider the human society as a system of highly interconnected units. Each unit will be characterized by two variables: its internal state and external state. This distinction is necessary due to the fundamentally nonlinear character of the real world. Indeed, almost any individual social unit is always unstable if it is left alone in a neutral environment. For example, it is well known that the two existing major socialeconomic systems are based on greed and envy, respectively. It means that a single unit left alone will never be satisfied and if its status is described with one variable only, it will grow indefinitely. In mathematical terms this is equivalent to a 
positive feedback loop. In reality, however, the external resources are always limited. Therefore, even in case of a very strong positive feedback, the status of the unit experienced by the environment (output state or external state) must remain within limits, whatever high the unit's level of activity (input state or internal state) is. This phenomenon is called saturation of the input-output characteristics. The internal state of the unit is measured by means of its excitation level, its external state may represent, e.g., its wealth, power, influence, etc.

Each unit interacts with a large amount of other units via mutual interconnections. These interactions between the units will determine the current status of the society as well as its dynamical behavior. Thus, we represent a social structure as a network (Wellman and Berkowitz, 1988). Due to the practical importance of the problem (only a stable society can survive), we are especially interested in the problem of social stability.

Our model then can be described by the following system of differential equations

$d x_{i} / d t=\sum_{j=1}^{N} a_{i j}(t) x_{j}(t)+I_{i}(t) \quad(i=1,2, \ldots, N)$

where $x_{i}(t)$ and $x_{i}(t)$ are the internal and external states of the ith unit at time $t, N$ is the number of units, $a_{i j}(t)$ is the strength of connection between the output of the $i$ th unit and the input of the ith unit (it is also a function of time) and $I_{i}(t)$ is the influence applied to the input of the ith unit from outside of the system.

The connection strengths $a_{i j}(t)$ form an $N * N$ connection matrix. The matrix elements are functions of time and they can be positive or negative according to the nature of influence of one unit toward the other. If the interaction between two units is 
positive, it means that one unit reinforces the other's activity (cooperation). In case of a negative matrix element one unit tries to suppress the other's activity (competition). Thus, we are dealing with a cooperative-competitive network. Such networks have been extensively studied by brain researchers (Grossberg, 1988), therefore our present approach can be called a neural network model of human society. We would like to emphasize that our model does not require any symmetry: a particular unit may be quite cooperative towards another one while the latter is hostile towards the former (see the relationship between Social Democratic and Communist parties in the past).

The correspondence between the unit's external state $x_{i}$ and its internal state $x_{i}$ is expressed by the relationship

$$
x_{i}=f\left(x_{i}\right) \quad(i=1,2, \ldots, N)
$$

where $f$ is a function that conveniently represents the saturated input-output characteristics. In this work we shall use the hyperbolic tangent function

$f\left(x_{i}\right)=\tanh \left(x_{i} / x_{T}\right)$

whose value tends to reach +1 at large enough positive and negative values of the argument, respectively. Here $\mathrm{x}_{\mathrm{T}}$ is a positive parameter that determines the speed at which the saturation values are reached: for large values of $x_{T}$ a greater value of $x_{i}$ is needed to reach saturation than for small values of $\mathrm{x}_{\mathrm{T}}$. As we shall see later, this parameter plays the role of 'social temperature'.

Evidently, the sign of the external state determines whether 
the unit's wealth, power or influence is positive or negative. A zero value means that the given unit plays a mediocre role in the society. The saturation values of \pm 1 correspond to total power and total loss of power, respectively. In case of political parties, e.g., $x_{i}=-1$ means that nobody votes for the ith party. It is quite possible that a number of units reach saturation: this case corresponds to sharing power with each other.

For very small values of $\mathrm{x}_{\mathrm{i}} / \mathrm{x}_{\mathrm{T}}$ the hyperbolic tangent function can be replaced with its argument. If, in addition, we assume that the elements of the connection matrix are just constant numbers, then we arrive at a strongly simplified society described by a linear model. As we have already mentioned it, reality is always nonlinear, but we can draw some conclusions even from this primitive model.

The stability of linear systems can be investigated by simple mathematical methods. One has to form an N Nth-degree algebraic characteristic equation on the basis of the connection matrix. If every real part of its roots is negative, then the solution of the system of linear differential equations is stable. The tedious task of finding the roots can be avoided by using other methods, e.g. the Hurwitz criteria, etc. (Ashby, 1960).

It is easy to understand the stability of some societies in terms of such linear systems of differential equations. Stability is the result of coordination between the units of the society. It is a property of the entire system and cannot be assigned to any part of it. It is entirely determined by the structure of the connection matrix. It is quite possible to form a stable system from unstable units and vice versa. The same units may form a stable system if they are joined one way and an unstable one if they are connected another way (Ashby, 1960). On the other hand, an extremely stable society can be built from both very unstable 
or very stable units (see, e.g. the Soviet Union under Stalin and Brezhnev, respectively).

A single linear unit $\mathrm{x}$ is represented by the simple equation

$d x / d t=a_{11} x$

where $a_{11}$ is a positive number that represents the connection between the 'output' of the unit and its own 'input', i.e. a simple positive feedback mechanism. The solution of this differential equation is an exponential function: the state of the unit steadily grows with time and the rate of growth is rapidly increasing.

It is amazing that in the real world such greedy and envious units can live together and even survive for considerable amounts of time without blowing everything up if they are interconnected with each other and with some 'benevolent' units characterized by negative internal feedbacks. It turns out that if the condition of $\left(a_{11}+a_{22}+\ldots+a_{N N}\right)<0$ is satisfied, it is possible to form a stable system from mostly unstable units.

At this point we shall abandon the linear aproximation and return to the nonlinear model. Since in our model the inputoutput relationship (3) is used, the external status of any unit will always stay inside an $\mathrm{N}$-dimensional hypercube defined by the extremal values of \pm 1 at saturation (Hopfield, 1984). Moreover, in most cases the system will tend to occupy the corners of this hypercube at equilibrium reached after a considerable amount of time has passed. The time needed to reach one of these equilibrium states depends on the 'social temperature' $\mathrm{x}_{\mathrm{T}}$ : the higher this temperature the slower the equilibrium is reached (see Figure 1). 
What does a social equilibrium mean in physical terms? One of the most general laws of nature is Boltzmann's minimum energy principle. The principle states that for a system in thermodynamic equilibrium the probability $\mathrm{p}_{\mathrm{A}}$ to occupy a state with energy $W_{A}$ is proportional to $\exp \left[-W_{A} /(k T)\right]$ where $T$ is the temperature and $\mathrm{k}$ is a universal constant called Boltzmann's constant (Feynman, Leighton and Sands, 1965). It means that at a fixed finite temperature the probability exponentially decreases as the energy increases, i.e. the system will occupy the state with the lowest available energy. At higher temperatures it is more probable that the system will occupy a state with a higher energy than it is at a lower temperature. In the extreme case of infinitely high temperature all finite energies can be reached with equal probability while in the other extreme case of a temperature at absolute zero the probabilities of all states with nonzero energies are zero.

The minimum energy principle governs the physical world around us. At low temperatures any physical system will always tend to occupy a state with the minimum available energy. This explains such phenomena as the the formation of crystals or the functioning of semiconductor devices. In the authors opinion this principle has a fundamental importance for social phenomena as well. Indeed, if we define 'social energy' as a quantity inverseIy proportional to the degree of satisfaction of the society as a whole, it is quite easy to represent social equilibrium as a state of the society with a local minimum of this 'social energy'. The stability of differential equations can be investigated by means of Liapunov functions that are extensions of the energy concept (La Salle and Lefschetz, 1961). For systems of differential equations with certain kinds of connection matrices one can construct Liapunov functions that have a possibly large number of 
equilibrium points corresponding to local energy minima (Grossberg, 1988). The time evolution of the physical system described by the differential equations seeks out these minima and stops at such points (Hopfield, 1984). The phase space of the system variables will then be divided into a number of regions of attraction. Depending on the initial conditions, the system will end up in one or another energy minimum. It can get out of such a state only if the temperature is high enough. This is equivalent to shaking the system, or waking it up. After this treatment the system will automatically find another energy minimum. It turns out that by first raising the temperature and then gradually cooling it down, one can even approach the absolute minimum of energy. This procedure is called simulated annealing (Kirpatrick, Gelatt and Vecchi, 1983). It is important to note that the energy is a quantity defined by the interconnections of the system and not by the individual units.

In order to appreciate this model, one should first consider the simplest non-trivial case of a 'society' consisting of two units only. We assume that the units are political parties that fight each other for power. The simulations that follow were done by the use of the DESIRE simulation software (Korn, 1988). The results will be presented in the form of graphs.

We shall start with connection matrices that are constant in time. Figure 1 shows how the external states $x_{1}$ and $x_{2}$ change in time for the simple symmetric connection matrix $a_{11}=a_{22}=1$, $a_{12}=a_{21}=-1$ (both units are equally 'gready' and both try to suppress the other's activity with equal efforts). The initial value of the internal state of the second unit is taken as zero: $x_{2}(0)=0$. The initial condition for the first unit is $x_{1}(0)=1$. Three pairs of graphs are shown in the figure with $a_{T}=0.25,1$ 
and 5, respectively. The value of the temperature parameter strongly influences both the initial values and the time history of the external states: lower social temperatures correspond to larger initial values and to faster approaches of the eqilibrium states. One can see that in this case the equilibrium state is $\mathrm{X}_{1}$ $=1, \mathrm{X}_{2}=-1$ : the first party gains absolute power over the second one.

The results are sometimes more convincing if we present them as phase plots, $i$. e. showing one external status $\left(X_{2}\right)$ as a function of the other $\left(\mathrm{x}_{1}\right)$. On these graphs one can clearly see the formation of the stable equilibrium points that correspond to energy minima: phase plots with different initial conditions all converge at two such points with coordinates $(-1,1)$ and $(1,-1)$, respectively (Figure 2 ).

The situation drastically changes if the units abandon their selfishness. Figure 3 presents phase plots for the connection matrix $a_{11}=a_{22}=a_{12}=a_{21}=-1$. There are no concentrated equilibria in this case: each initial condition leads to a separate state of equilibrium. Note that the equilibrium points are all situated along a straight line in the phase plane.

Interesting features appear in non-symmetric cases. The connection matrix for the case shown in Figure 4 has the following elements: $a_{11}=a_{22}=a_{21}=1, a_{12}=-5$. (The first party supports the second one but its sympathies are strongly rejected.) Phase plots are shown for four different initial conditions: $x_{2}(0)=0$ and $x_{1}(0)=0.2,0.4,0.6$ and 0.8 , respectively. The behavior of the system is quite complicated and we find that three corners of the two-dimensional "hypercube" appear as equilibrium points: for the first case the coordinates are $(-1,1)$, for the second case they are $(1,1)$, for the last case we have $(1,-1)$. In the third case, when $x_{1}(0)=0.6$, the system does not 
appear to settle down at any corner at all. This can be seen from the time history shown in Figure 5.

Naturally, social units do not behave in such a rigid way as it is represented in the preceding examples. They learn from their mistakes and modify their behavior accordingly by changing their connections to the other units. Figure 6 shows a ten-year history of a two-party system. It starts with the connections $\mathrm{a}_{11}$ $=a_{22}=1, a_{12}=5, a_{21}=-1$ (the second party strongly supports the first one which, in turn, tries to mildly suppress its rival). After about three years it seems that the policies of the second party were right. Since its popularity initially decreased, its support of the first party lead to the latter's decline. At the end of the fourth year, however, the first par$t_{y}$ 's popularity begins to grow. Then the leadership of the second party abruptly reverses its course: it starts to strongly oppose the first party. As a result, the first party quickly loses all of its popular support and the second party wins.

The model is even capable to simulate dramatic social changes when an already established equilibrium is destroyed and a new power struggle leads to a different equlibrium. Such a situation is demonstrated in Figure 7. The relationship of the two parties is represented by the connections of $a_{11}=a_{22}=1, a_{12}=$ $a_{21}=2$ (both parties support each other). As a result, a power sharing equilibrium is quickly established. At this point both parties reverse their policies and start to oppose each other (as it frequently happens in real life). This results in an initial decline of both parties' popularity, but eventually the first party wins and the second disappears from the political arena. The above examples demonstrate our thesis that social changes can be explained (and to some extent even predicted) on the 
basis of redistributions of the connections between the units. These changes take place more or less rapidly according to different 'social temperatures'.

The model becomes more realistic and interesting as more units are added. As an illustration, the time history of a fiveparty system is shown in Figure 8. The connection matrix is

$a=\left|\begin{array}{rrrrr}1 & -1 & -1 & -1 & -1 \\ -2 & 1 & -1 & -1 & -1 \\ -1 & -3 & 1 & -1 & -1 \\ -1 & -1 & -4 & 1 & -1 \\ -1 & -1 & -1 & -5 & 1\end{array}\right|$

All units are equally greedy and all of them try to suppress all the others. However, four of them are especially hostile to one party each and their degree of aggressiveness is different. The result is a wavy power struggle with two winners and three losers.

\section{CONCLUSIONS}

The model presented in this paper is based on two fundamental principles: 1) The dynamic behavior of a complicated society is determined by the connections between its units. 2) The society has a tendency to settle down at some equilibrium states that minimize a certain 'social energy' at given 'social temperatures'. It takes more time to reach an equilibrium at higher temperatures but it is easier to change established equilibrium states if the social temperature is higher.

We do not pretend that our model can predict the future of the world but it can be quite helpful for analyzing the consequences of certain attitudes in complex social environments. For example, the connections between different political parties can be quantitatively measured by the number of times they vote together in a parliament or congress. It has been established 
that electoral swings happen when these connections change (Nannested, 1988). It is then relatively easy to establish the time dependence of the connection matrix for a multi-party political system, analyze its history and draw conclusions about the reasons of past political events. It is the author's intention to carry out such an analysis for a European country in the near future. Similarly, relationships between nations can be treated by the use of our model. It can also be helpful for the development of future political strategies at different levels.

\section{NOTES}

This work is supported by the National Science Foundation under Grant ECS 8700849 and by the Danish Research Council for Natural Sciences.

The author is indebted to Professor G. A. Korn for his generous permission to use the simulation software DESIRE in this work. The stimulating discussions with Professors B. Mayoh and P. Nannestad are much appreciated. 
Ashby, W. R. 1960. Design for a Brain, London: Chapman \& Hall. Feynman, R. P., Leighton, R. B., \& Sands, M. (1965). The Feynman lectures on Physicg. Reading: Addison-Wesley.

Forrester, J. W. 1972. World Dynamics, Cambridge, MA: WrightAllen Press.

Grossberg, S. 1988. Nonlinear neural networks: principles, mechanisms, and architectures. Neural Networks, 1:17-61.

Hopfield, J. J. 1984. Neurons with graded response have collective computational properties like those of two-state neurons. Proceedings of the National Academy of Sciences, 81:3088-3092. Kirpatrick, S., Gelatt, C. D. and Vecchi, M. P. 1983. Optimization by simulated annealing. Science, 220:671-680.

Korn, G. A. 1988. Interactive dynamic system simulation and Mathematics on personal computers. New York: McGraw-Hill.

La Salle, J., \& Lefschetz, S. 1961. Stability by Liapunov's direct method. New York: Academic Press.

Nannestad, P. 1988. Personal communication.

Wellman, B. and Berkowitz, S. D. (eds). 1988. Social Structures: A Network Approach. Cambridge: University Press. 


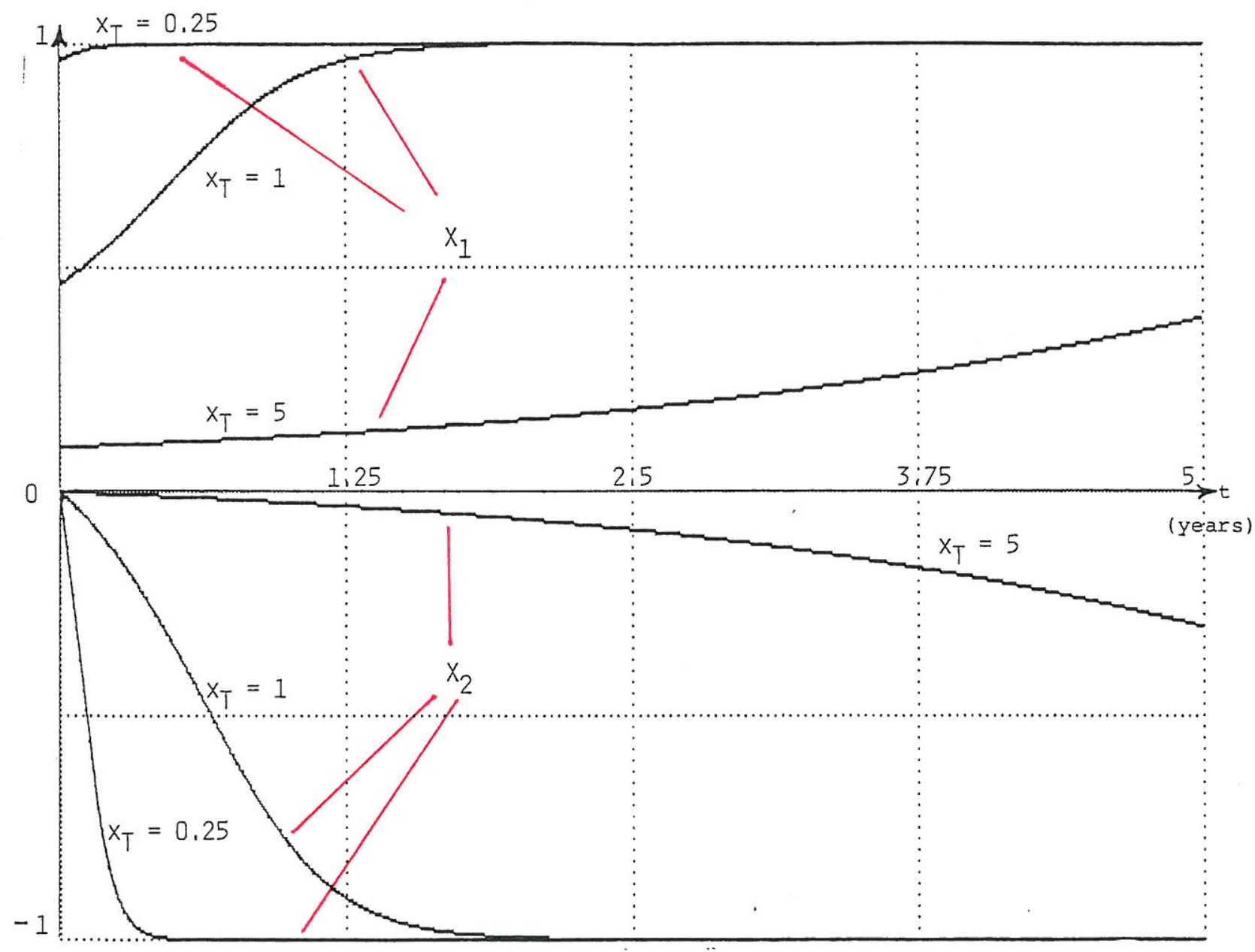

Figure 1.

The time history of a two-party system with the connection matrix $a_{11}=a_{22}=1, a_{12}=a_{21}=-1$ for three different values of the social temperature $\mathrm{x}_{\mathrm{T}}$. 


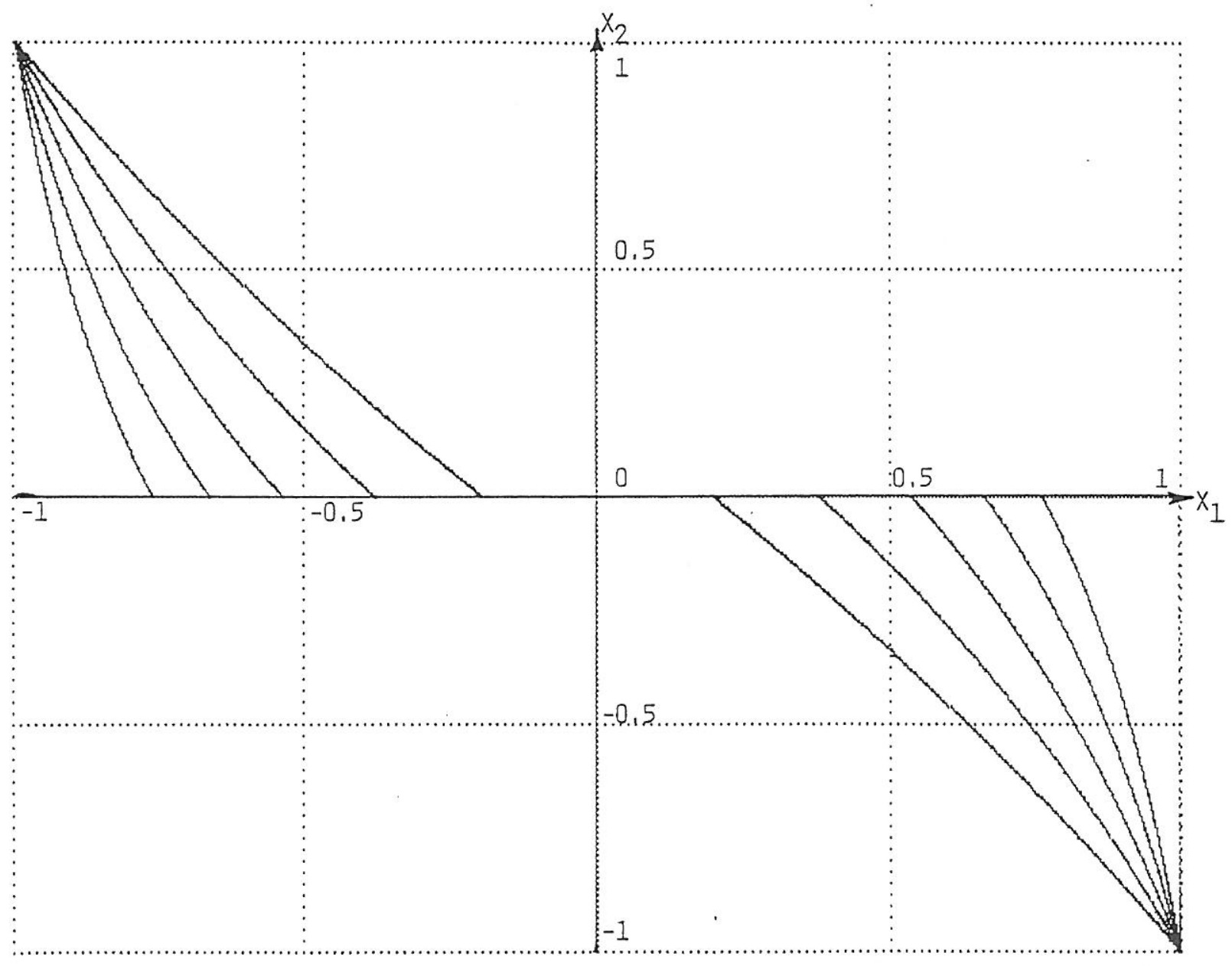

Figure 2.

Phase plots for the two-party system shown in Figure 1 with different initial conditions. 


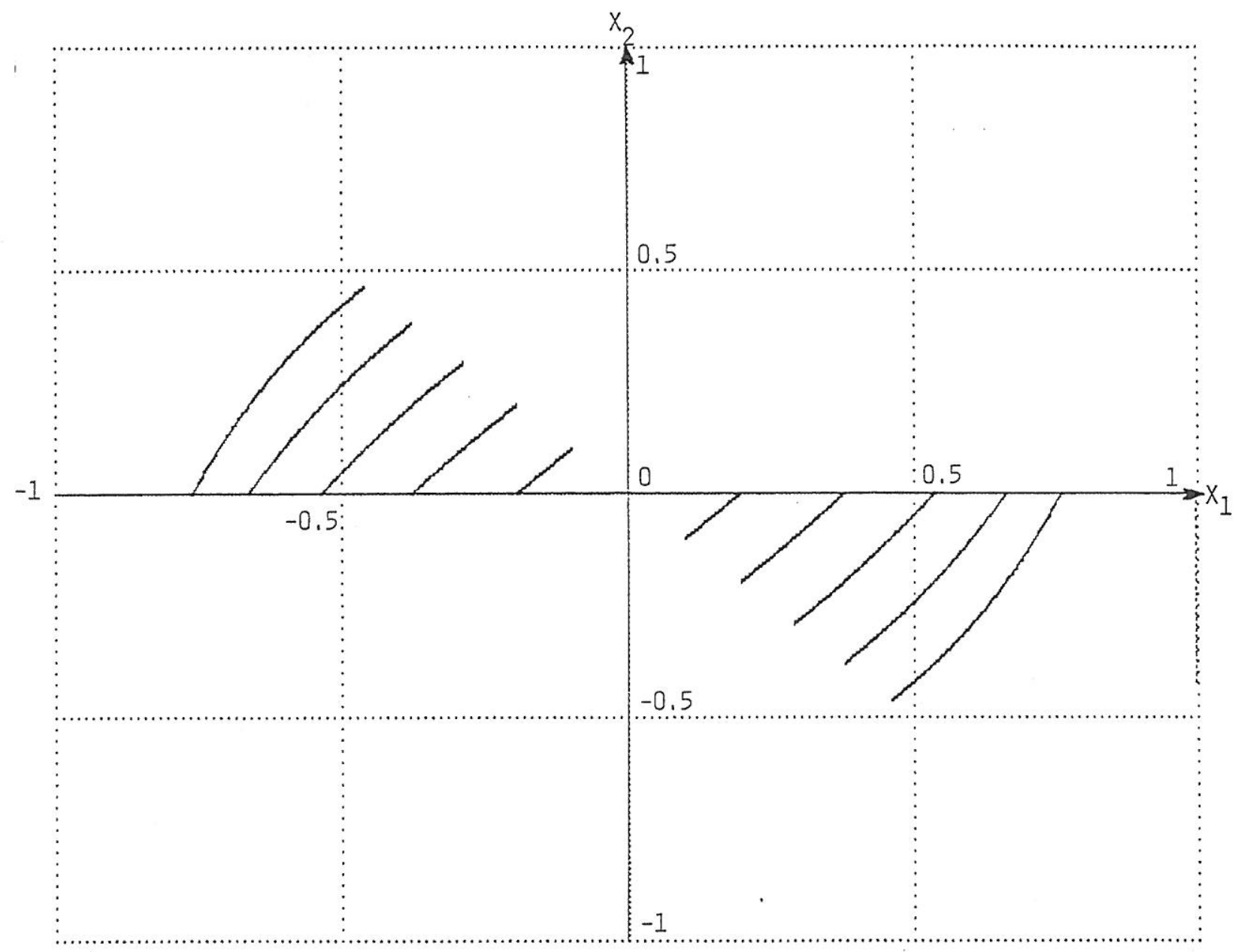

Figure 3 .

Phase plots for a two-party system with the connection matrix $a_{11}=a_{22}=a_{12}=a_{21}=-1$. 


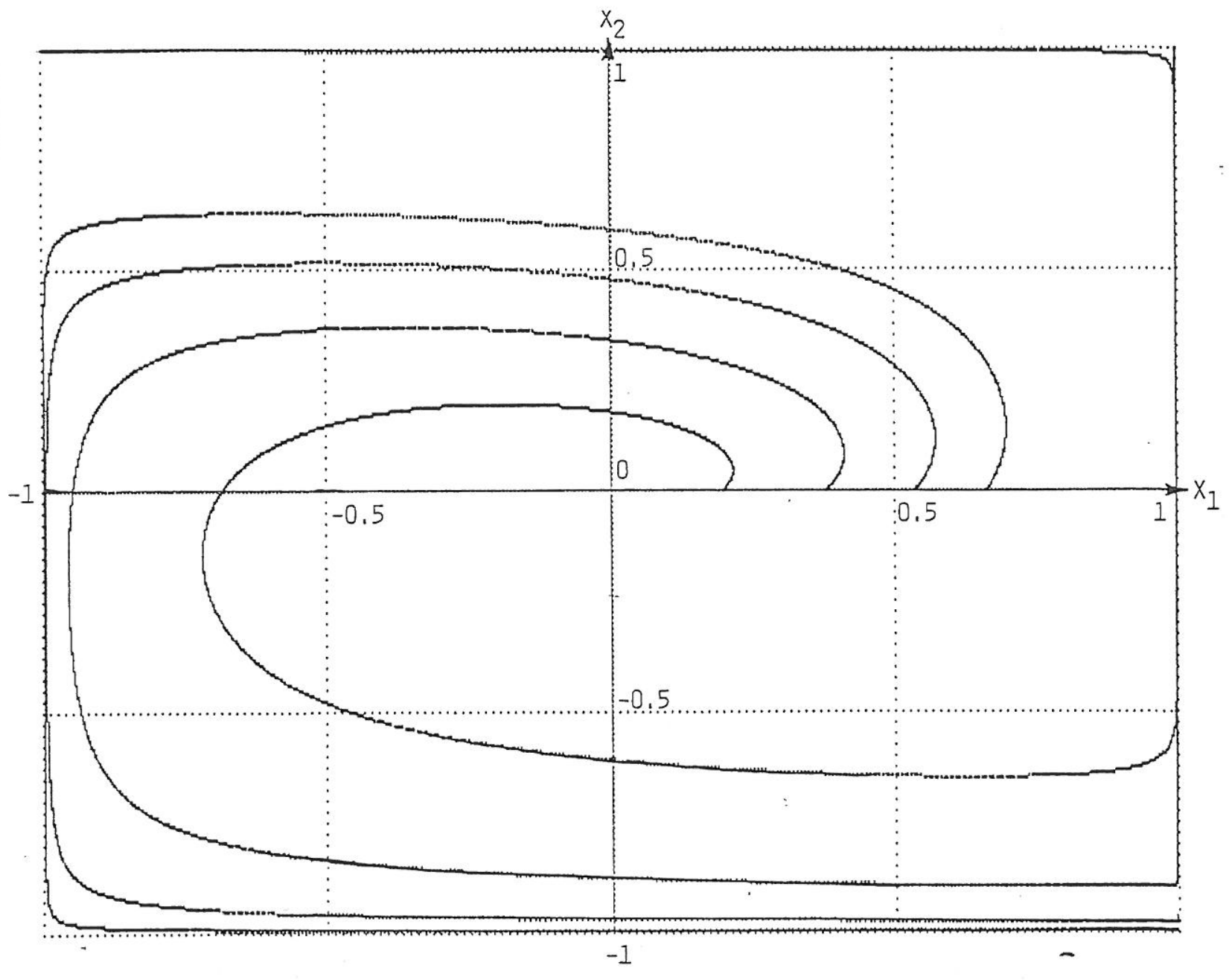

Figure 4 .

Phase plots for a two-party system with a highly asymmetric connection matrix. 


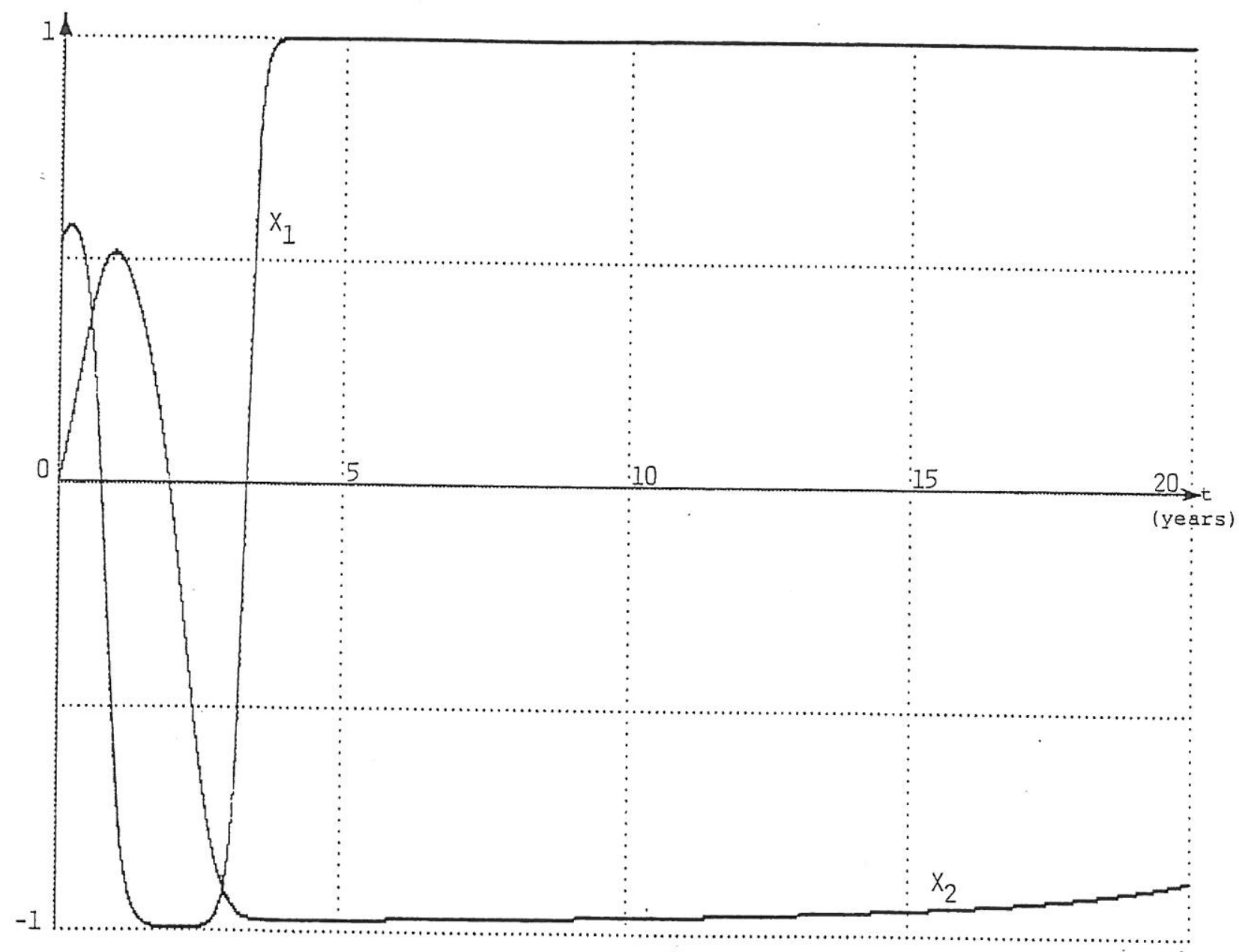

Figure 5 .

Time history of the two parties represented in Figure 4. 


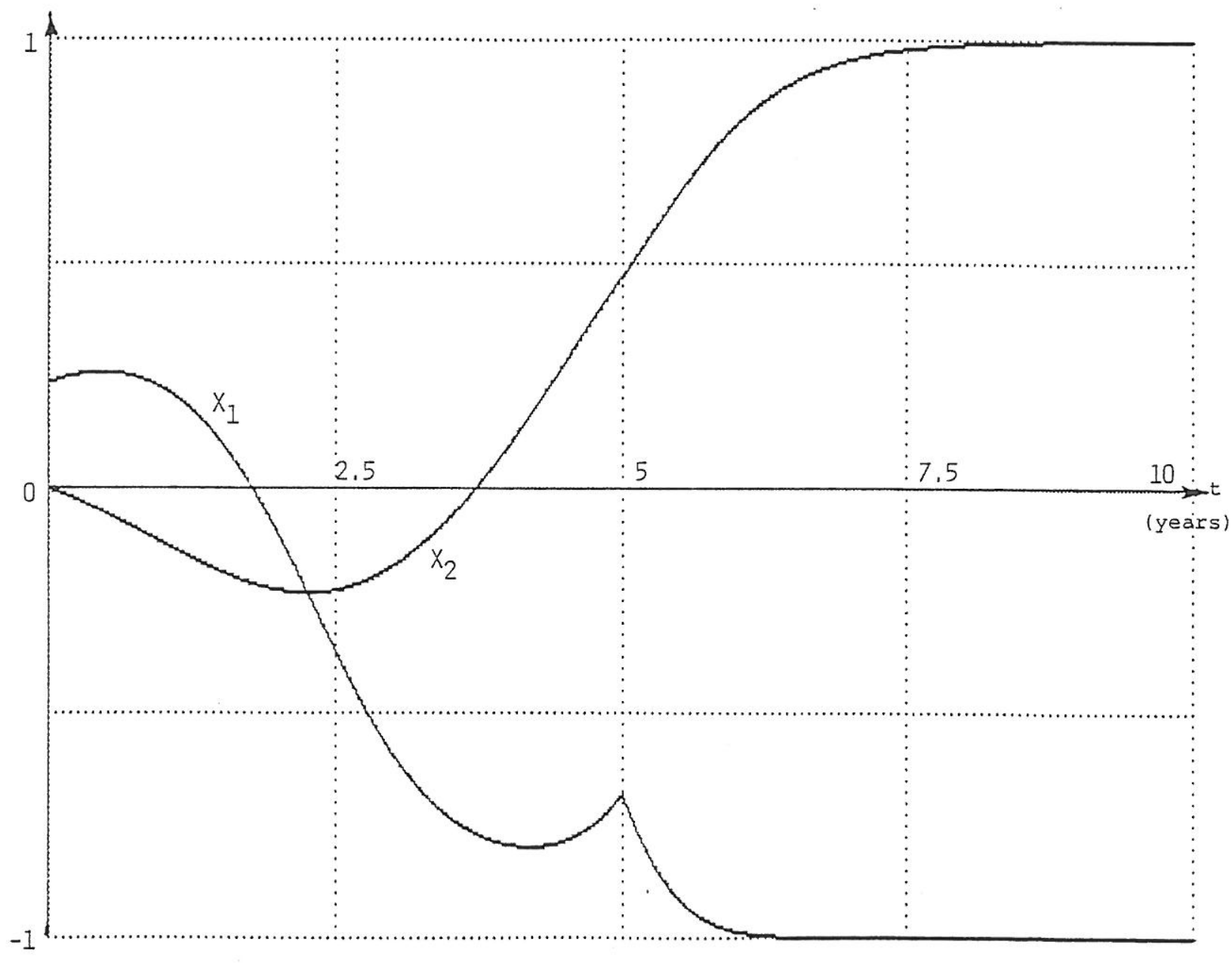

Figure 6.

Time history of a two-party system with a policy change. 


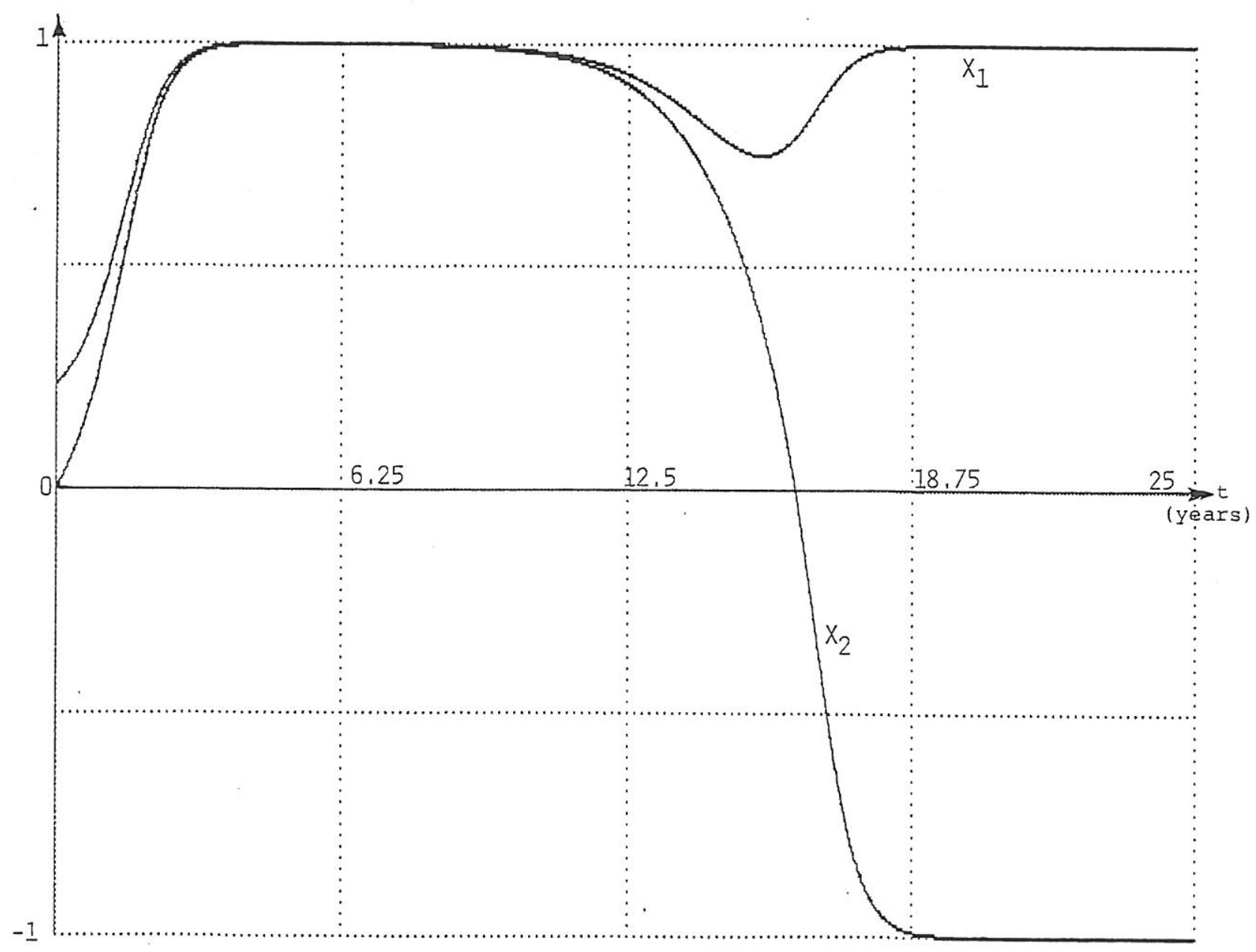

Figure 7 .

Destruction and re-establishment of a social equilibrium. 


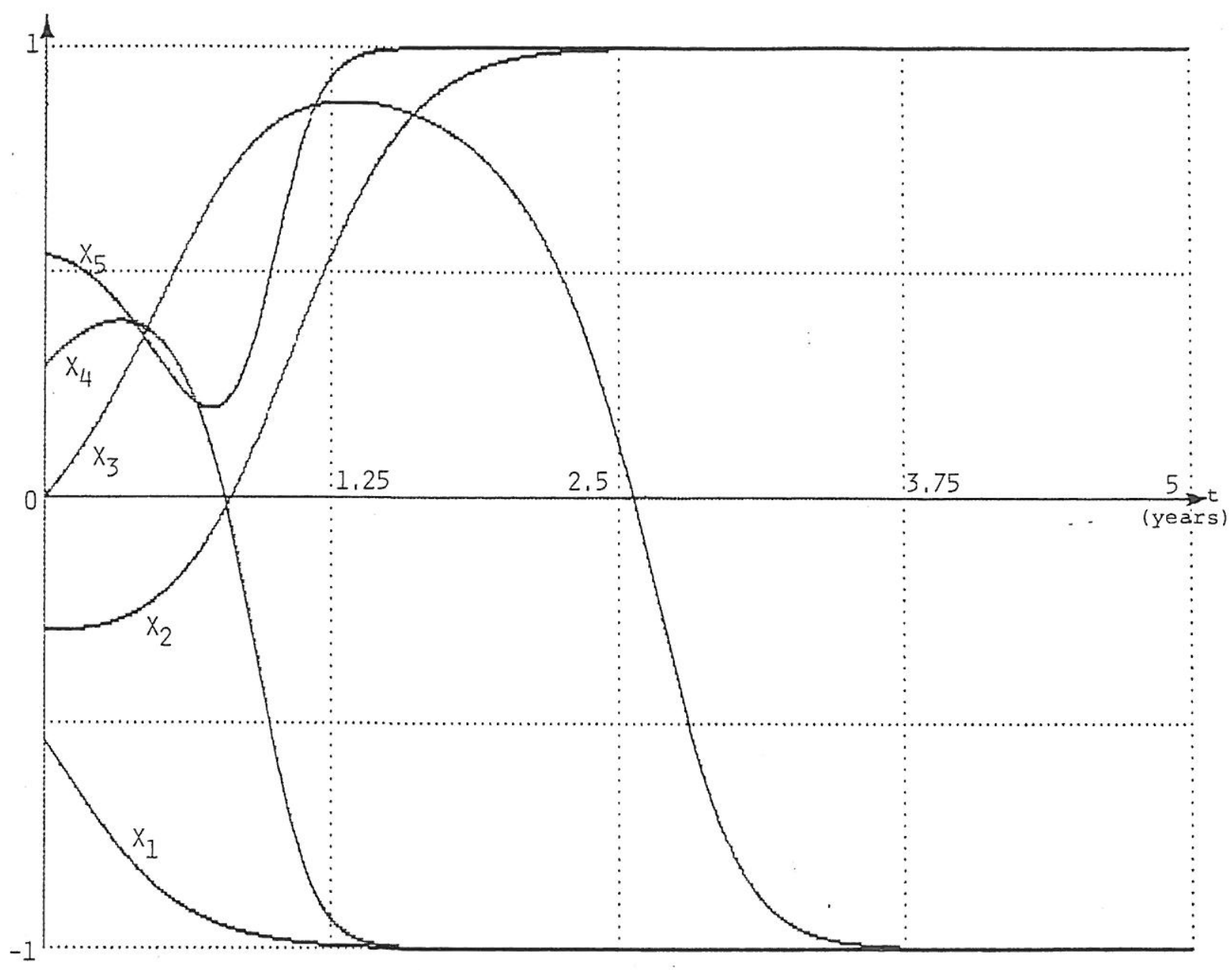

Figure 8 .

Time history of an asymmetric five-party system. 


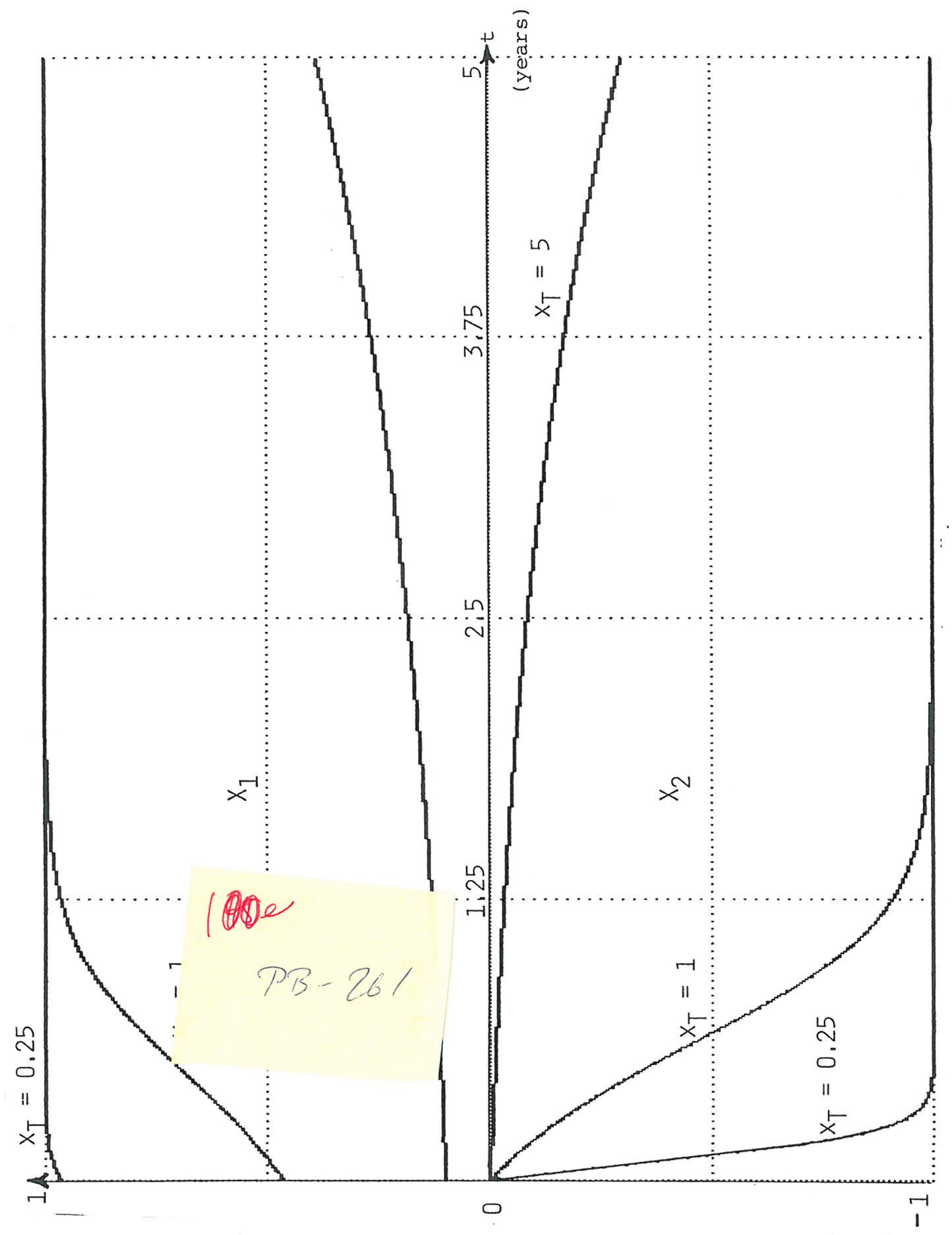

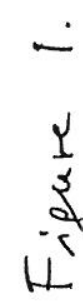




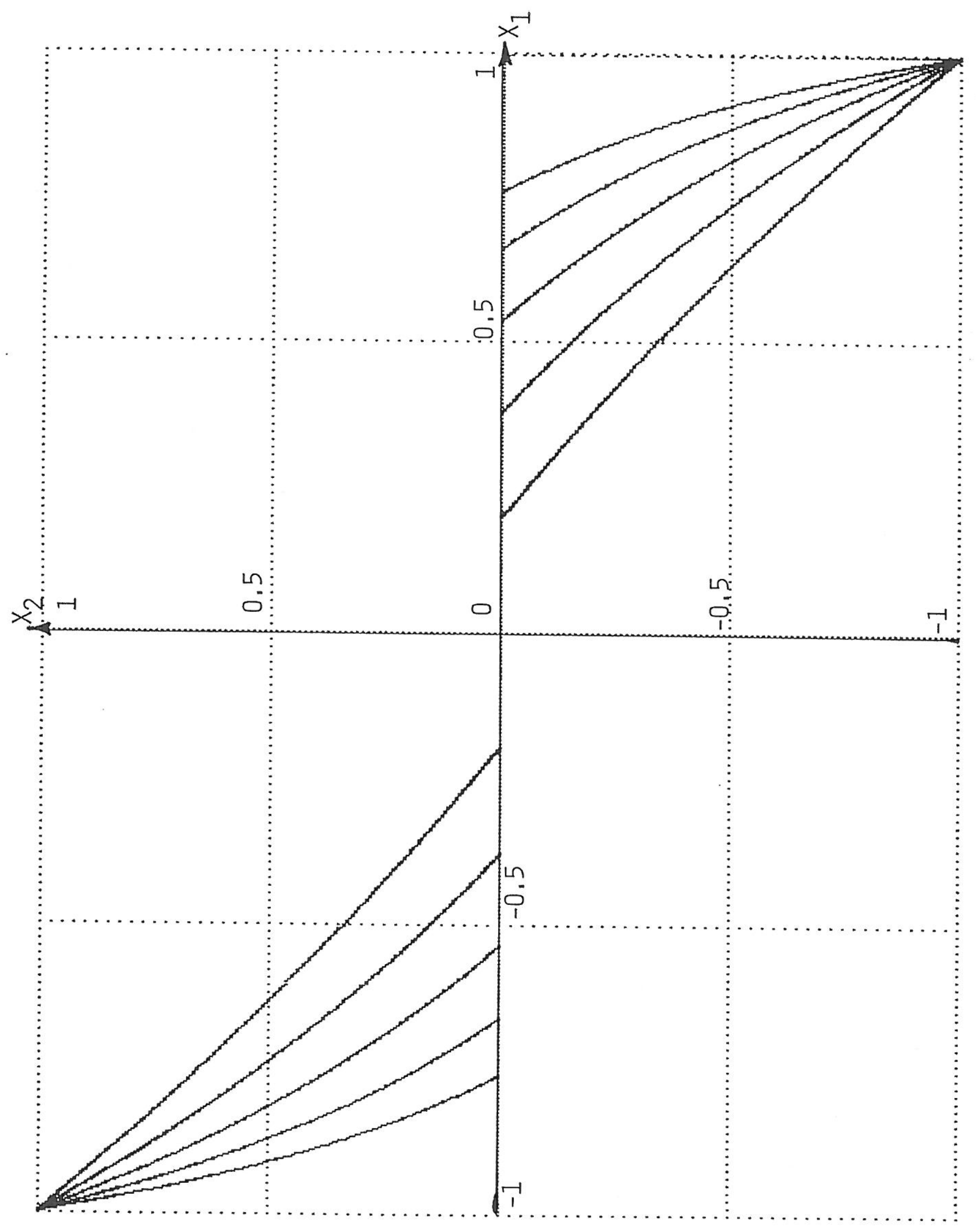

$n$
9
$\vdots$
$\ddot{L}$

$\xi$

$\vdots$
$\vdots$
$\vdots$
$\vdots$
$\vdots$
$\vdots$

$\frac{1}{3}$ 


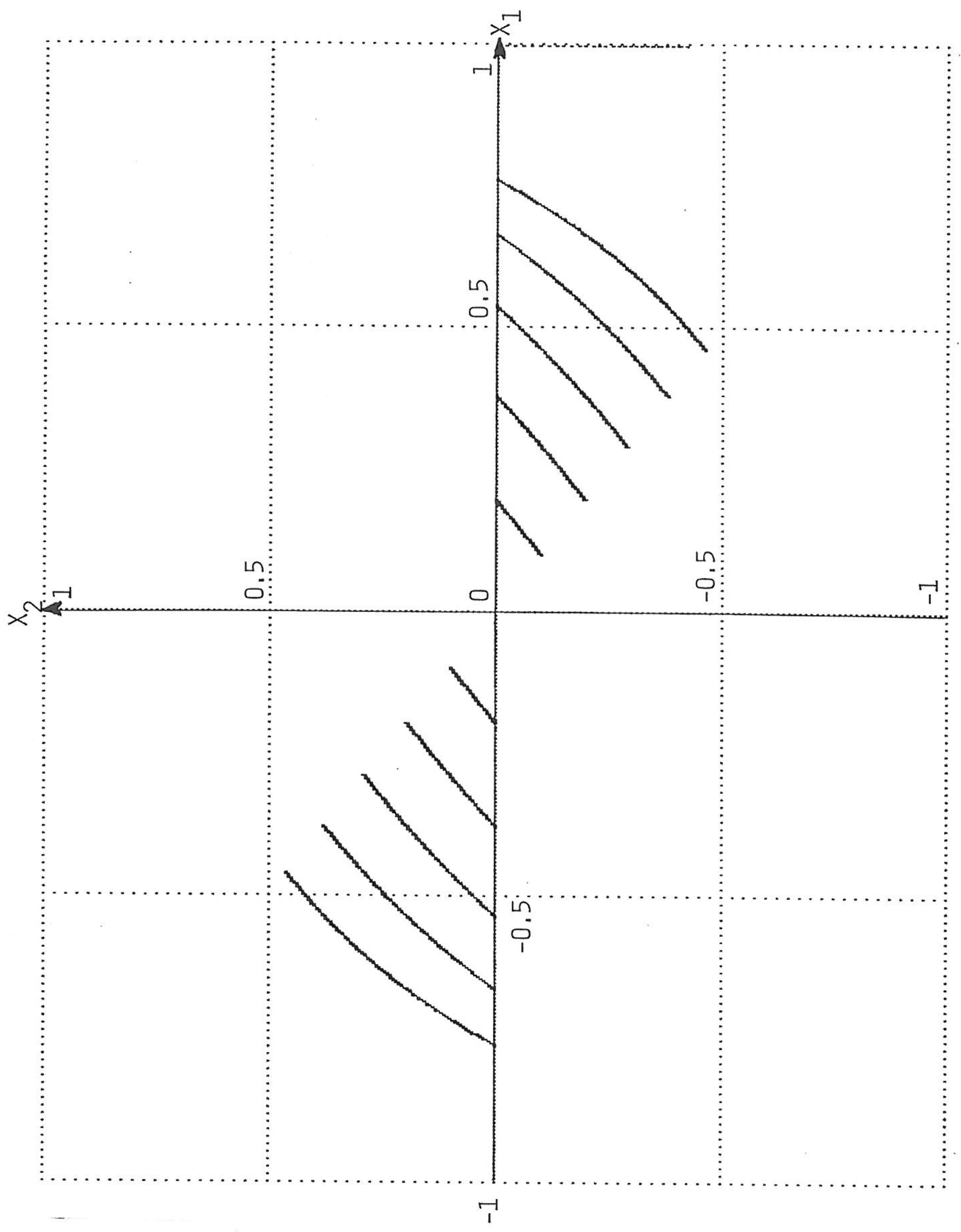

$N$
$\xi$
$\vdots$
$d$

$\vdots$
$\vdots$
$\vdots$

$Y$
5
$\vdots$
$\vdots$
5
5
5

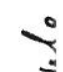

$e^{5}$ 
<smiles>CC1CC#CC1I</smiles> 


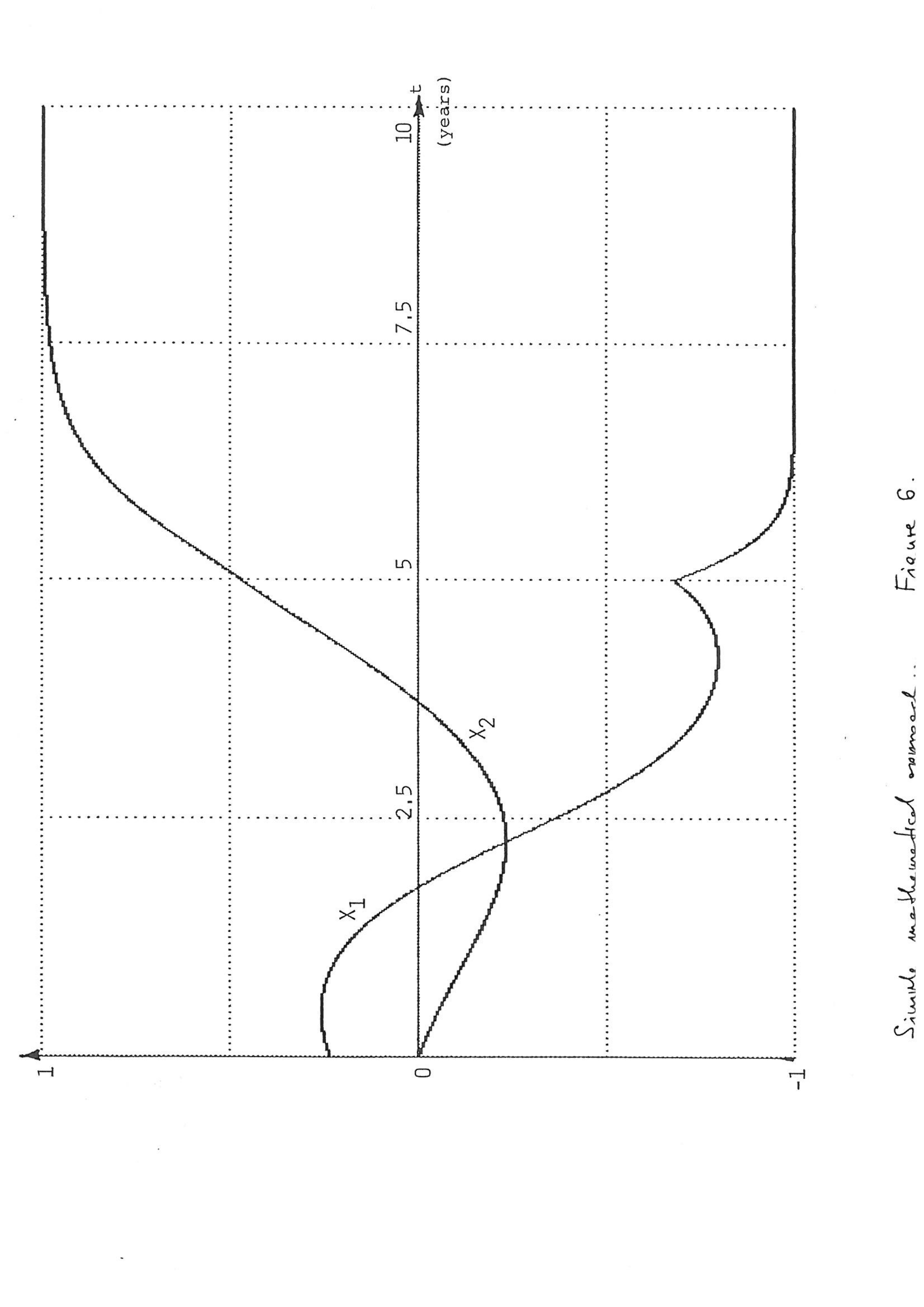




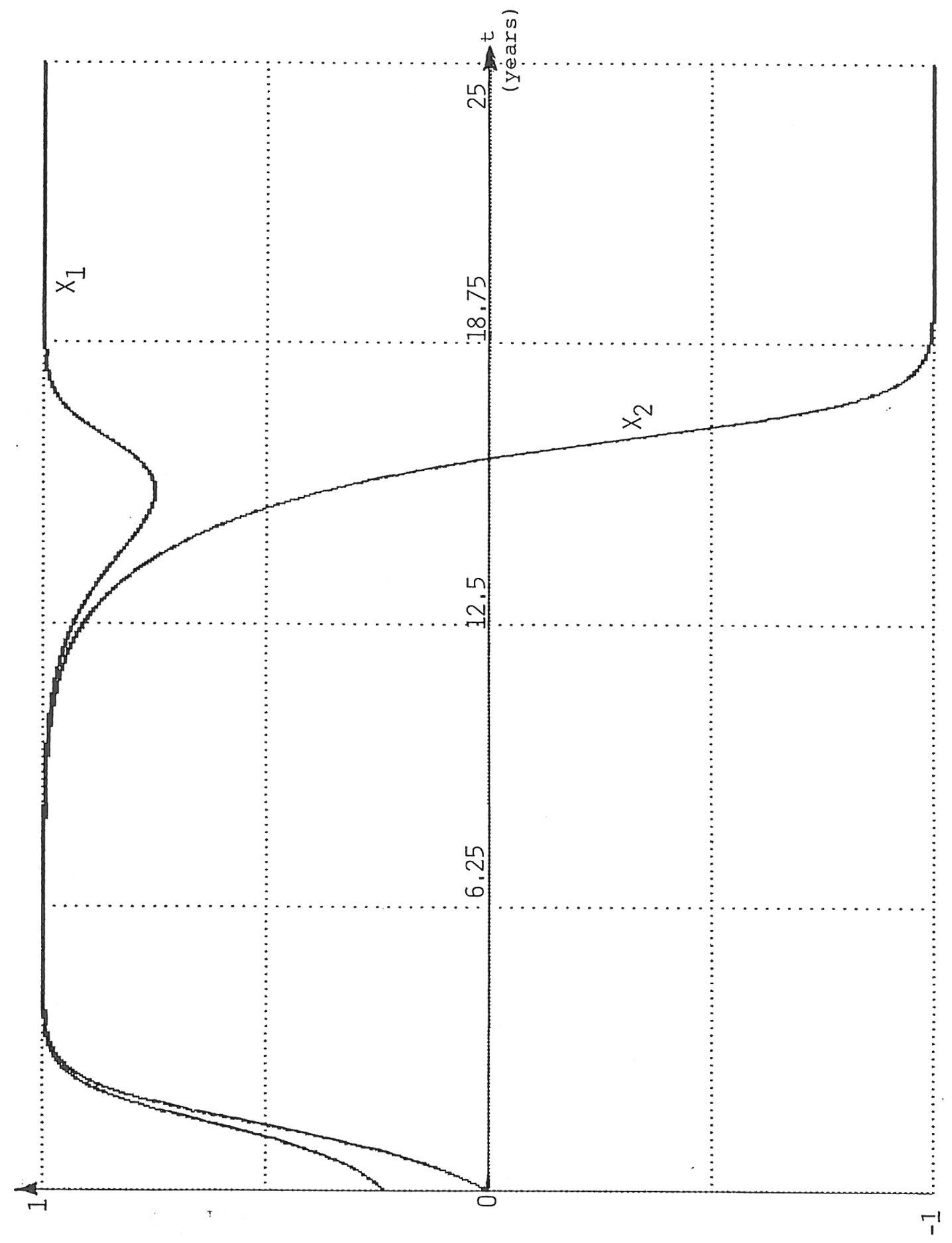




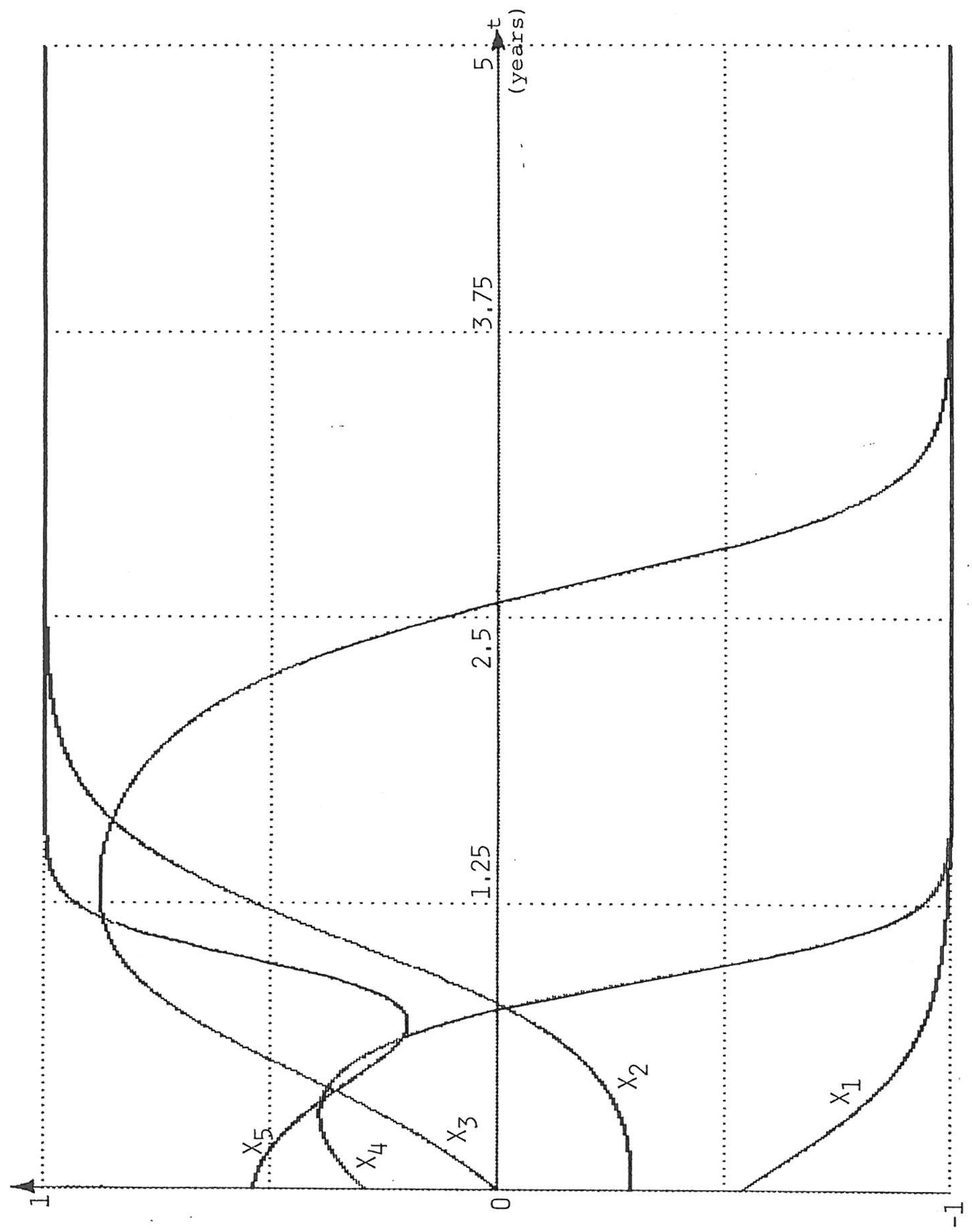




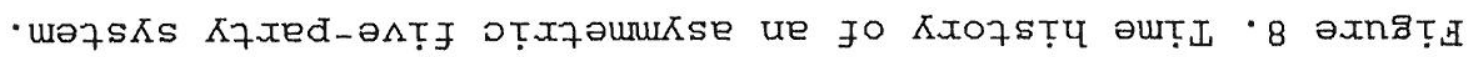

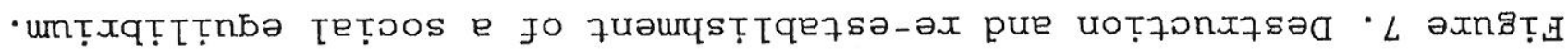

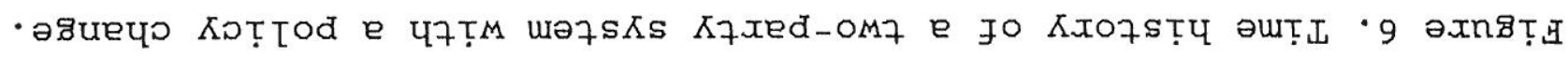
•

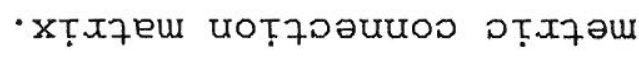

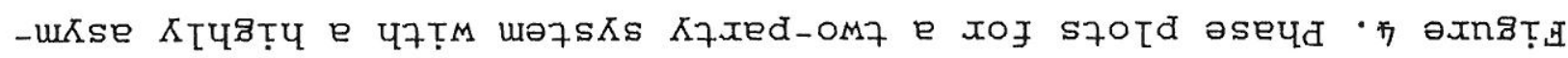
$\cdot I-=I Z_{e}=Z I_{e}=Z Z_{e}=I I_{e}$ xṬxpur

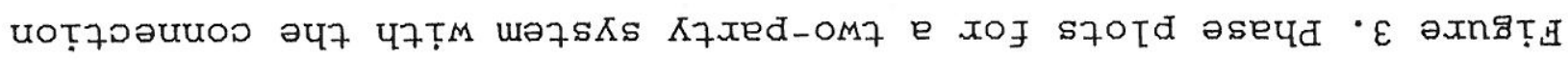

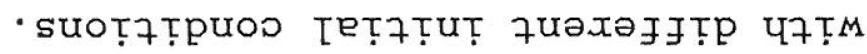

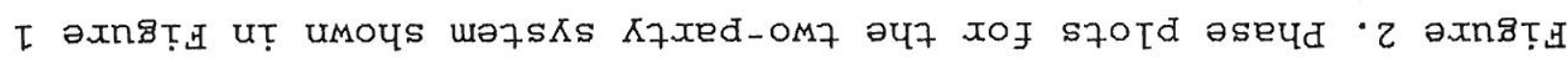
. $山_{X}$ әxnұexəduəz [eт̣рos әч7 jo səntes

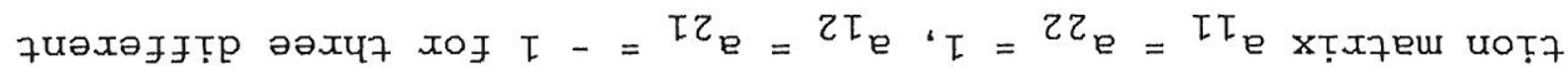

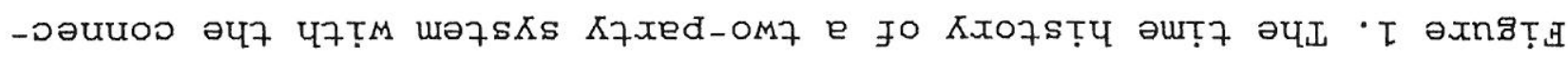
SNOILdHD GHกDIH 